\title{
Improving the Usability of Collaboration Methods and Technologies in Engineering
}

\author{
Lars Wolter ${ }^{1}$, Haygazun Hayka ${ }^{2}$, and Rainer Stark ${ }^{2}$ \\ ${ }^{1}$ Chair of Industrial Information Technology (IIT) \\ Department of Machine Tools and Factory Management, Technische Universität Berlin \\ lars.wolteratu-berlin.de \\ ${ }^{2}$ Division Virtual Product Creation \\ Fraunhofer Institute for Production Systems and Design Technology (IPK) \\ rainer.stark@ipk.fraunhofer.de
}

\begin{abstract}
This paper illuminates certain needs and solutions in collaborative engineering like the ability to initiate collaboration sessions without preparation steps, the acceptance of collaboration solutions due to heterogeneous IT landscapes and the protection of intellectual property rights. These problems are observed together with current solutions in commercial products and research projects. An overview of those solutions is presented, including their workings, problems and current state of development adding some in depth information about current research work conducted by the authors to address these problems.
\end{abstract}

Keywords: Collaboration, collaborative engineering, PLM, heterogeneous IT landscape, intellectual property rights.

\section{Introduction}

The industry sets new requirements for collaborative engineering due to technological improvements on products and product development methods, increasing complexity of supply chains and the trend to establish virtual teams. The paper discusses these requirements and the resulting fields in need of action. Each field shows different opportunities for the industry. Technology can give better integration and usability, the processes can be more transparent and standardized and the human factors can receive more attention to increase the stimulation of the stakeholders. To utilize all the opportunities in these fields, the collaborative engineering processes, methods and tools can not only focus on the technical goals and how to achieve them, they must also consider the human factor, containing the other stakeholders and there reasons. Only then the task work can be united with teamwork for successful collaboration.

\subsection{The Need for Collaborative Engineering}

The increasing complexity of consumer products and industrial goods also increases the complexity of their development. This is given by a raising number of parts in 
each individual product and especially the combination of multiple engineering domains into a single product. Additional complexity in today's product development originates from release cycles that need to get shorter to stay competitive. To address the complexity a company needs to involve more people in the development process, each being an expert in his domain. This also includes the engineers which are experts for specific domains, parts, functionalities or steps in the development and manufacturing process.

As with common meetings collaboration is a task that does not produce anything but needs to be done to be successful. Therefore the collaboration needs to be efficient and natural for all participants. The collaboration in product development is happening on many different levels starting from asynchronous groupware systems, telecooperation solutions, viewing collaboration and full featured interactive collaboration. Additionally the collaboration can be done locally or across long distances, it can be with or without the use of digital tools. Then there is also the difference of collaboration inside companies which happens intensively and the collaboration with partners and suppliers which is performed less intensively [1] and is also shown in figure 1. This increases the complexity of collaboration management which only needed to supply meeting rooms and a telephone number to allow collaboration. To manage all the collaboration scenarios in companies today, additional people need to be involved being experts in the area of collaboration.

The focus during this paper lies in engineering scenarios during virtual engineering. It does not address collaboration during concept development, production or selling.

\section{How much time take the following activities of your daily working time (estimated proportion of time)}

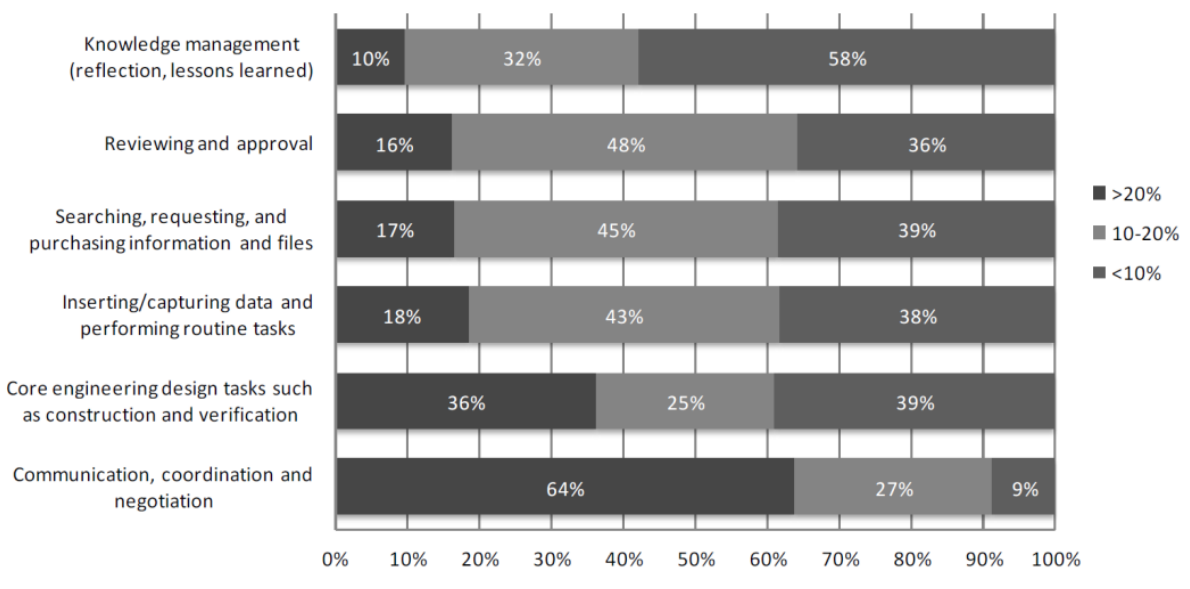

All Industries $(n=1401)$

Fig. 1. Distribution of Engineering Working Time [1]. Communication, coordination and negotiation happen intensively in the industry. 


\subsection{Problems of Collaborative Engineering}

In collaboration there is a large field of problems that can be analyzed and addressed. This article focuses on collaboration supported by digital tools that an engineer uses during his product development tasks. For an engineer the types of tasks have changed over time. There is much more information needed to fulfill his day-to-day tasks forcing him to spend a lot of his time to search and acquire this information, not only from IT-Systems but also from other colleagues or partners. This also means he has to supply information he generates during his work either by storing them in an IT-System or by directly communicating them to other people. This additional overhead is already part of the collaboration happening in a company. Looking at basic collaboration tasks without the use of digital tools already introduces a lot of problems. If you leave someone a message on a post-it it can get lost during cleaning, your block of post-its could be empty or you try to find someone for a talk but don't know the room where that person currently is. Those problems can be directly translated to collaboration using digital tools. For example the email getting lost in the spamfolder, no free space on the network drives or the Web-Ex session that cannot initiate because of a too restrictive firewall. These kinds of problems can be addressed very effectively by rules and a good organization, which are necessary to achieve robustness in using digital engineering technology [2]. But digital tools for collaboration introduce new kind of problems that need to be addressed separately.

\subsubsection{Heterogeneity}

Due to the increasing fragmentation of companies, either through outsourcing or by integrating other companies leads to the use of multiple IT-Systems dedicated to the same kind of task. This is also true for the collaboration tools, either stand-alone or integrated, because most of them can only be connected to tools of the same vendor. This is not a problem with the telephone for example; it can be used with every other telephone from different vendors.

\subsubsection{Tool Acceptance}

An engineer today has to use multiple digital tools to solve his engineering tasks, this includes CAx, PDM, ERP, MES, Excel, Outlook and various other tools. The number of tools increases in a collaboration situation because an extra collaboration tool needs to be used. Same goes for engineering tools, which normally only the collaboration partner uses. This is a frustrating situation for the engineer because it maybe a tool he only uses irregular when contacting a supplier for example and sometimes differs from his regular tools in usage and even methodic concepts. The engineer is naturally blocking to use this extra tool for collaboration resulting in reduced efficiency or lesser collaboration.

This also happens for local collaboration scenarios, where multiple people discuss a situation with a specific tool. This happens in design reviews for example, where a dedicated operator is necessary to operate the tool introducing an extra layer into the interaction. 


\subsubsection{Protection of Intellectual Property}

The ideas of new products as well as the methods and processes to produce them are a constant interest for all competitors in the market. Therefore this knowledge needs to be kept secret. Collaboration using digital tools is normally associated with sharing your own digital data. This results in trust problems with any kind of new or specialized tool which connects multiple stakeholders for collaborative purposes. Opposite to that generally used tools like emails have a very high trust even if they are used insecurely. The problem is to make any kind of collaboration tool used be trusted to protect the intellectual property; otherwise it cannot be effectively used.

\subsection{How Are These Problems Currently Addressed}

The market has a multitude of solutions to achieve collaboration in engineering. The system vendors of larger software products started to integrate collaboration features in their products. This includes features common to current social media products like text, audio and video chat combined with the sharing of product data. One example of those solutions is 3D-Live in Catia V6 from Dassault-Systemes. Such a system works very well in a homogenous environment, in which all participants use the same CAD program which tightly integrates into the PDM of the same vendor to make the product data sharing available for collaborative engineering. Using a different CAD system breaks all the collaboration features.

This problem can be solved by deploying all applications to every user, forcing the engineer to use multiple IT-Systems in collaborative scenarios. Another way to address the heterogeneity problem is to use stand-alone collaboration solutions. Standalone solutions are a less complex extra piece of software, but also need a context switch and the conversion of the data from the engineering tool. Multiple vendors developed standalone tools to allow collaboration without the need of supplying the whole authoring tool set to all users.

There are two kinds of stand-alone solutions for collaboration. Screensharing solutions are the easiest to use in this category and don't need any data conversion. They work by capturing the contents of the screen or a specific application window and transmit that as a video stream to the other participants. These solutions are well known in web conferencing and are widely used because of their ease of usability. The user just starts the screensharing solution and decides which screen to share. After the setup, he only operates his preferred tool. Products in this category are WebEx from Cisco or WebConf from Adobe. But the concept of screensharing lacks the possibility of an equal participation in the collaboration scenario, because only one participant can present his screen to all others. Even when using multiple monitors, a monitor for each participant would be needed to present all the different views.

The second type of solution is applications that use their own visualization engine to display geometric models. Most of those solutions import data from the engineering tool and share them across multiple participants. Because these tools are separate from the authoring tools, they normally only import common exchange formats. This means the collaborating engineer needs to export the product data from his application in a suitable format for the collaboration tool and all the data need to be 
transmitted to all participants. This conversion and transfer of product data induces long setup times. This kind of tools can have a lot of functionality to alter the viewing, leaving annotations and therefore support the collaboration very well, but requires in additional tool-knowledge for the engineer. Changes that can only be done in the authoring system would need an export of the changed product data and redistribution of those changes to all participants.

The most basic way of collaboration thru sharing data is handled asynchronously. Data management solutions allow locking of complete files by different users but there is no technique available that can merge two differently changed CAD files like Microsoft Word is offering for documents. Most PDM and other data storage systems ease the asynchronous collaboration with large files by duplicating them to different sites making them rapidly available from different locations. This is still limited in speed, but is already well established among the industries.

The last kind of well-established collaboration solutions consists of web based groupware solutions. They are often based around whole communication solutions for email, task and workflow management. They allow the users, to exchange tasks, documents and other information using wikis forums or blogs. Newer systems also incorporate so called social office functions to allow commenting, rating and sharing of information across the company intranet. Examples for these kinds of systems are Microsoft Office with Sharepoint and Outlook or open source groupware solutions like Liferay, Tiki-Wiki or other portal solutions. These solutions are independent of product development but can be customized to fit specific products and companies. The type of collaboration is limited, but due to the inclusion of real time communication and web based editors for documents these solutions are not only used for cooperation but also full collaboration.

\subsection{Research to Increase the Collaboration Efficiency}

Some research is done to support the engineering collaboration. One approach is to let different CAD systems communicate with each other, to allow collaborative design even with heterogeneous CAD systems. One of those approaches in [3] uses a common set of commands. Every CAD system translates its own authoring commands to this common set which is distributed to all participants and at each target platform converted to a command of the specific CAD system. Therefore it does not need to exchange any product data beforehand. This approach allows concurrent design from the beginning; it does not allow modification of existing product data.

Other Research is done to allow better network communication even in firewalled scenarios across companies for communication applications [4]. This is a very fundamental type of research affecting nearly all collaboration attempts which communicate thru the internet. Also very fundamental is research to resolve conflicts in collaboratively authored CAD. This is addressed for example by research activities to integrate Boolean operations in CAD systems [5].

There is also research activity to enhance distributed design reviews. There are solutions that adapt to different kind of devices allowing the use of large VR-Cave-Systems together with participants only using desktop computers during the collaboration. One 
such approach is documented in [6]. The setup they are using uses VRML models to visualize 3D data but can also show the screens of the participants using screensharing.

Many research projects focus on the protection of the intellectual property. Everything related to encryption is only protecting the data while it is traveling between the collaboration participants. If the aim is to not let one of the participants misuse data from other participants, any encryption is of no use. Therefor some research projects describe methods and algorithms to watermark geometric models [7]. This way it can be traced back where copies originated allowing the owner to sue them. Other methods reduce the details in certain areas by using multiresolution meshes and specifying the detail priorities of certain areas [8].

On the other hand there is also research going on for 3D model reconstruction from image sequences. With flaws this was possible 1996 from camera images [9]. Better algorithms, the good quality of the rendered images and faster computers allow much better reconstruction [10]. But for all reconstruction algorithms it is important that there is at least one image from every feature necessary to reconstruct the original model. If the back side or inside of an object is never shown, it cannot be reconstructed.

\subsection{Areas in Need of Action}

\subsubsection{Technology}

The technology for collaboration is in a very good state, but there are technological problems when different solutions need to be connected.

The rapid increase in information and the expectation of its global availability introduces a new field of information management that does not require a central distribution point but intelligent information containers that can manage the containing information and is able to route those information to systems and participants in a collaborative scenario that need them.

Besides the technical areas that are in need of action, the collaboration between engineers needs to address human factors to make the collaboration to something an engineer wants to do instead of something he needs to do. This can be seen in interaction with tools, where a user is pleased to use a tablet-pc to read its newspaper using simple gestures. This kind of technology common to consumer products needs to be adapted to solutions for the industry.

\subsubsection{Processes}

The business processes need to address the specialty in collaborative processes. When working in a collaborative manner, each step has a meaning for the task the group does together, like the patterns in [11]. This can also lead to additional tasks for collaborative processes, because after a collaborative session the gathered information needs to be converged from multiple participants before it can be evaluated.

\subsubsection{Methods}

The different methods of collaboration can be as coarse has to choose from personal meeting, phone call or use of email to very fine grained methods that define the format of the 
emails to send for specific tasks. Having a method for handling specific collaboration tasks is a must, to ensure correct flow of the information.

\section{New Solutions for Collaborative Engineering}

To fill some of the gaps presented above the following solutions are described. These solutions handle different collaboration scenarios like local collaboration on multitouch tables as well as remote collaboration over the network.

\subsection{Using Current Touch Technology for Local Cooperation}

The technology for touch is well established in the consumer area. The technology is also very mature in its use. To be of a real benefit in the industry not only the technology needs to be used, but also the methods must adapt to the scenarios, where multitouch environments can be used to raise the efficiency of engineering processes. During the research at the Fraunhofer IPK methods where developed to visualize product structures on multitouch tables [12]. The requirement was a good usability with touch devices but also be understandable und usable in a multi-person-scenario where the participants have different views onto the multitouch device. This resulted in a Voronoi [13] based structure as seen in figure 1. This special structure was analyzed to see if it can fulfill some typical tasks in engineering like search and compare operations with this structure [14].

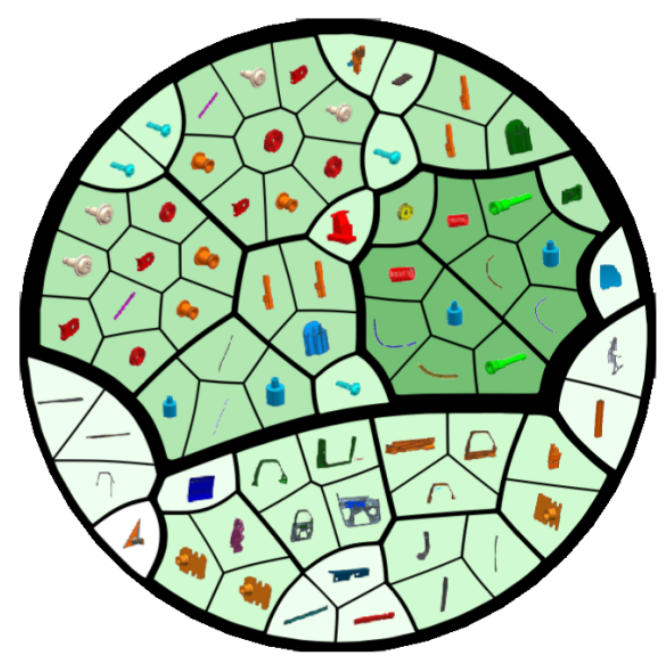

Fig. 2. Touch optimized visualization of a product structure 


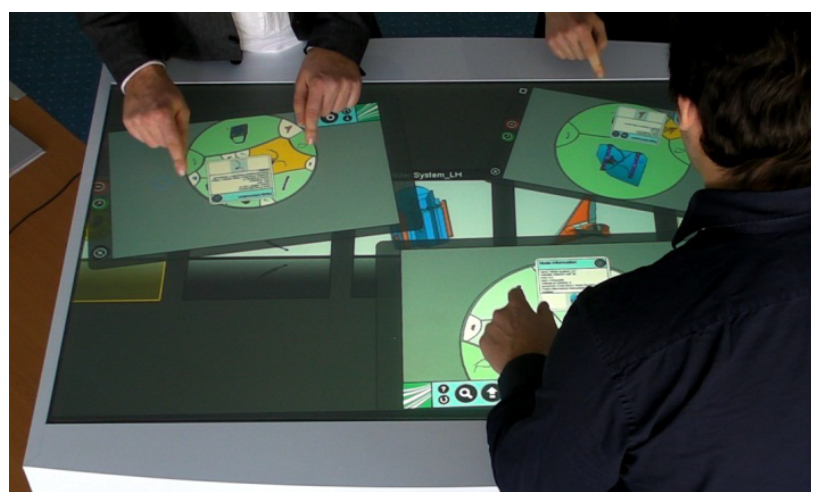

Fig. 3. Multi-User Multitouch environment for design reviews

In figure 3 this special application can be seen in a multi user environment. It allows multiple participants in a meeting to either work in their own workplace or to cooperate with some or all of the other participants. This allows part of the cooperating group to prepare their content while others are discussing a previous item. The example is the door of a car. In this example the expert for the door opener can discuss some details with the chassis expert while the experts for the window-liftersystem illuminate the use of an extra-ordinary expensive part to the management.

\subsection{Technology for Secure and Instant Collaboration}

The here presented solution constitutes a combination of screen sharing and the local visualization at each participant. In contrast to screen sharing not the whole program window or the whole desktop is being transmitted, but only the 2D image of the rendered 3D model, which is superimposed with the images of all participants. A correct superposition is necessary so that every participant can correctly perceive the visual impression of the complete product and properly interpret the correlations and distances between the components.

This collaboration technique focuses on different scenarios shown in figure 4. All participants shown in figure 4 see a 3D representation of the object being reviewed, in this case a truck. The parts in blue are locally existent as 3D-Modells and are locally rendered on the computer and the rendered image is transferred to all participants. The gray parts of the model do not exist on the local computer. They are just 2D images streamed from one of the other participants. All views share the same point of view and orientation while looking at the truck. This information is also shared among the users and consists of a simple matrix. The scenario can also incorporate special participants like the mobile lead engineer which only needs a web browser to join the session. He is not supplying any 3D model, he just consumes the images. The opposing case is the PDM System at site B which just renders it locally stored data and sends it to the others. This participant does not consume any information.

All the other participants, the OEM at site east and the two suppliers deliver their own data and consume from the others. The OEM holds the 3D models of the chassis, while supplier A holds the cabin and supplier B the wheels. They only deliver their 


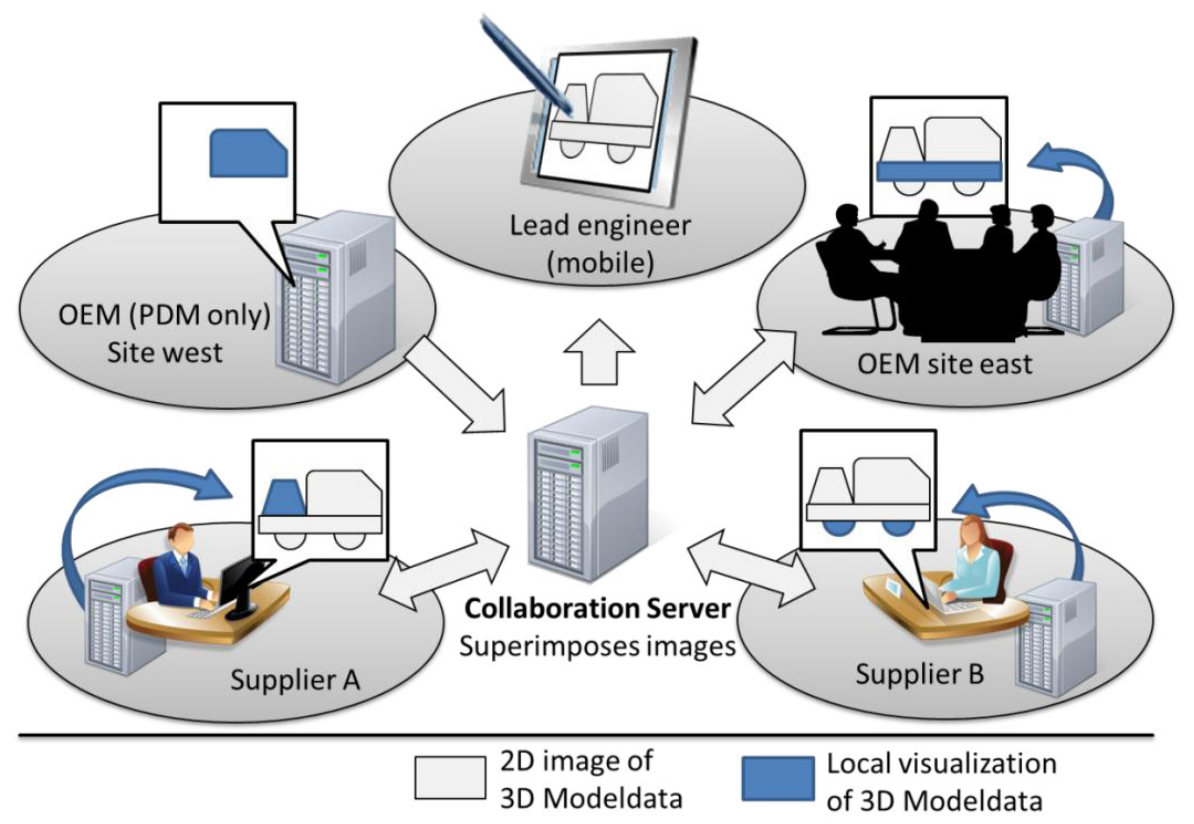

Fig. 4. The different collaboration scenarios condensed in a single collaboration

own property as images without being afraid that for example supplier B can steal the 3D model data from supplier A.

To achieve the correct superposition a so-called depth image is transmitted additionally. The depth images can be used, to decide for every pixel from which $2 \mathrm{D}$ image the respective pixel should be used (figure 5, bottom right).

To create, transmit or show it on a screen, a 2D image of a 3D model must be rendered. This process is called image synthesis, where for each pixel on the screen must be determined, which part of the 3D model it represents. The color of that point is being shown at this pixel (figure 5, bottom left). The depth image is constructed on the same principle. This is achieved by storing a distance value instead of a color value. If you interpret this distance value as a color, so is the value that represents the pixel brighter for larger distances and darker for shorter distances.

At multiple participants a color- and depth rendering is created that are all collected. These can now be used to construct a joint image. Condition is that all participants have the same point of view. This joint point of view acts as a zero point of the viewed scene and is reached by using the aforementioned matrix that is shared among the participants. This aspect corresponds to the technique of local visualization where likewise just the view matrix needs to be exchanged.

Assuming there are three participants with color renderings C1-C3 and depth renderings D1-D3. Then for each pixel it is analyzed which of the three Dx is the darkest. If it is D3 then the color value of C3 is being used. That way a new image is assembled where all models of all three participants are integrated. An interpolation allows combining images with different resolutions as long as the aspect ratio is maintained. 


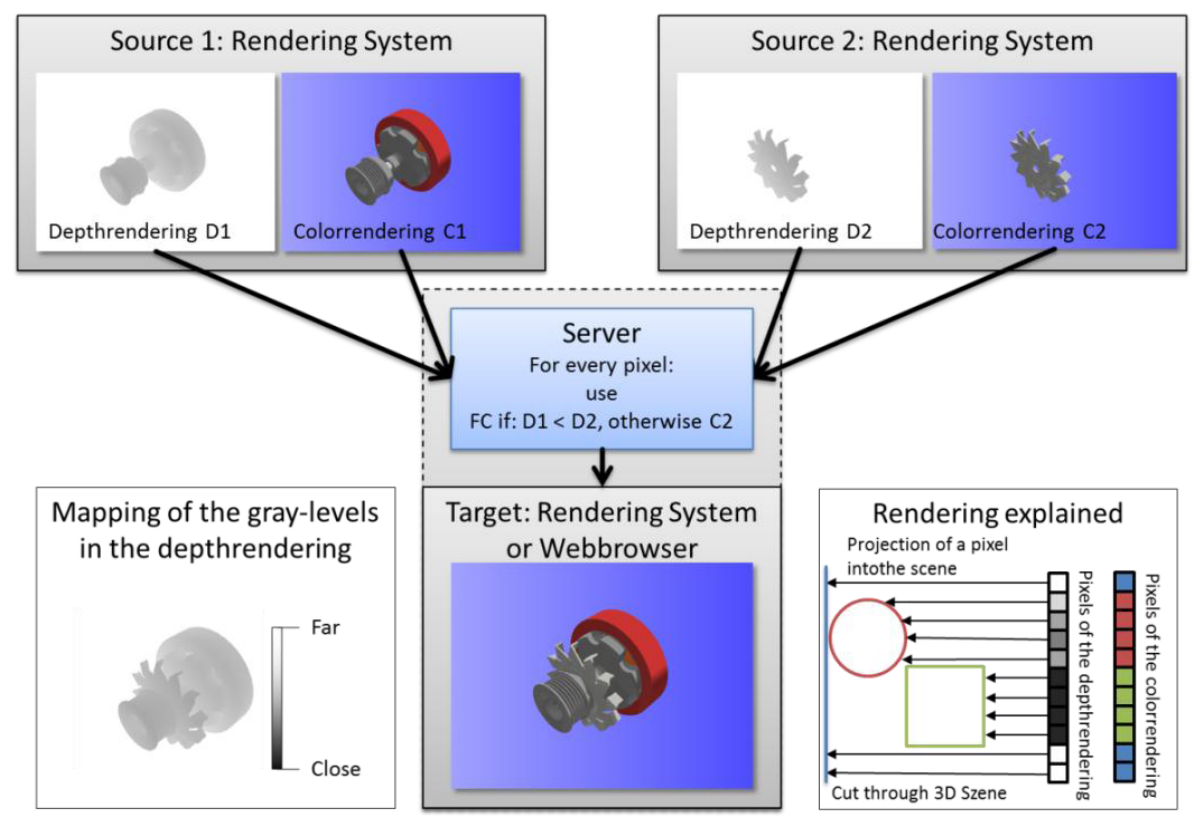

Fig. 5. Description of the image merging algorithm that merges two rendered images displayed at the top into a single image in the bottom middle. Therefor it uses a depth image explained on bottom left. Bottom right describes the basic principle of rendering a 3D scene into a $2 \mathrm{D}$ image in conjunction to the rendering of a depth image.

This is of advantage when generating images on hardware with different performances. The images from different sources can still be combined. The realization of this technology was executed in a prototypic collaboration tool.

\subsection{Increased Acceptance through Collaboration Using CAD Systems}

To approach the problem of acceptance, methods were evaluated to include collaboration functionality in existing CAD systems. Through the strong link with the rendering an easy connection via a plugin is difficult. In the course of work the CAD systems Spaceclaim and NX were extended via plugins in a way that they communicate their viewing location with other participants and could react to commands. Doing that the CAD systems showed limitations of different magnitude. The realization of the plugin proved more difficult for NX than for Spaceclaim. An automatic reacting e.g. to the network commands could not be realized with the NX API until the end of the project. Nonetheless the realization of the plugins proved that a collaborative coupling of two very diverse CAD systems is possible and a consequent commitment across manufacturers towards an open adaption of the API according to the $\mathrm{CPO}$ could create new possibilities.

Similar as in [3] it was also tested to fulfill some modeling tasks using heterogeneous CAD systems. But the target was different as in the paper because the focus is still located in the design review scenario where existing CAD Models need to be examined and possibly changed. Therefor the problem of identifying the same parts in the different systems needs to be managed. The research in this area is still ongoing, to deliver a solid solution. 


\section{Conclusions}

The increasing need for effective collaboration solutions is a challenge for today's companies. But system vendors and research facilities are continuing to generate better solutions. Useful cross vendor programs like the Codex of PLM Openness can ensure that future collaboration solutions will not suffer because of heterogeneous IT landscapes. The continuous evolvement of user interaction technologies from the consumer market into the industry gives the opportunities for user friendly and easy to use solutions. The presented solutions and ongoing research work introduce new accents for collaboration. These accents can be used by the industry to bring new concepts of collaboration into the companies to increase their collaboration abilities.

\section{References}

1. Müller, P., Pasch, F., Drewinski, R., Bedenbender, H., Hayka, H., Stark, R.: Study on Collaborative Product Development and Digital Engineering Tools. In: Rivest, L., Bouras, A., Louhichi, B. (eds.) PLM 2012. IFIP AICT, vol. 388, pp. 389-399. Springer, Heidelberg (2012)

2. Stark, R., Kim, M., Woll, R., Wolter, L.: The way forward of Virtual Product Creation how to achieve robustness in using digital engineering technology? In: 15 Seminario International de Alta Tecnologia, pp. 41-52 (2010)

3. Li, M., Gao, S., Wang, C.C.L.: Real-Time Collaborative Design With Heterogeneous CAD Systems Based on Neutral Modeling Commands. Journal of Computing and Information Science in Engineering 2007(7), 113-125 (2007)

4. Stark, R., Gärtner, H., Wolter, L.: Verteilte Design Reviews in heterogenen Systemwelten. ProduktDaten Journal 15(1), 45-49 (2008)

5. Zheng, Y., Shen, H., Xia, S., Sun, C.: Conflict resolution of Boolean operations by integration in real-time collaborative CAD systems. In: Meersman, R., Tari, Z. (eds.) OTM 2007, Part I. LNCS, vol. 4803, pp. 238-252. Springer, Heidelberg (2007)

6. Daily, M., Howard, M., Jerald, J., Lee, C., Martin, K., McInnes, D., et al.: Distributed Design Review in Virtual Environments. In: Churchill, E., Reddy, M. (eds.) Proceedings of the Third International Conference on Collaborative Virtual Environments (CVE 2000), pp. 57-63. ACM, New York (2000)

7. Kuo, C.-T., Cheng, S.-C., Wu, D.-C., Chang, C.-C.: A Blind Robust Watermarking Scheme for 3D Triangular Mesh Models Using 3D Edge Vertex Detection. Asian Journal of Health and Information Sciences (1), 36-63 (2009)

8. Zyda, M., Capps, M., Pausch, R., Bishop, G., Pojar, E., Schmalstieg, D.: User-controlled creation of multiresolution meshes. In: Proceedings of the 2003 Symposium on Interactive 3D Graphics, SI3D 2003, p. 127. ACM Press (2003)

9. Beardsley, P., Torr, P., Zisserman, A.: 3D model acquisition from extended image sequences. In: Buxton, B., Cipolla, R. (eds.) ECCV 1996. LNCS, vol. 1065, pp. 683-695. Springer, Heidelberg (1996)

10. Snavely, N.: Scene Reconstruction and Visualization from Internet Photo Collections: A Survey. IPSJ Transactions on Computer Vision and Applications 3, 44-66 (2011)

11. de Vreede, G.-J., Briggs, R.O.: Collaboration Engineering: Designing Repeatable Processes for High-Value Collaborative Tasks. In: Sprague, R.H. (Hg.) Proceedings of the 38th Annual Hawaii International Conference on System Sciences. IEEE Computer Society Press, Los Alamitos (2005) 
12. Woll, R., Wolter, L.: Kollaborativer Design Review am Multitouchtisch. In: Reiterer, H., Deussen, O. (Hrsg.) Mensch \& Computer 2012 - Workshopband: Interaktiv Informiert Allgegenwärtig und Allumfassend!?, pp. 439-443. Oldenbourg Verlag, München (2012)

13. Balzer, M., Deussen, O., Lewerentz, C.: Voronoi treemaps for the visualization of software metrics. In: Proceedings of ACM Symposium on Software Visualization, pp. 165-172 (2005)

14. Schulze, E.E., Wolter, L., Hayka, H., Röhlig, M.: Intuitive Interaktion mit Strukturdaten aus einem PLM-System. In: Konferenzband des 10. Gemeinsames Kolloquium Konstruktionstechnik, pp. 71-87 (2012) 